\title{
Rol del bloqueo interauricular avanzado en la predicción de accidentes cerebrovasculares isquémicos.
}

\author{
Rocío del Pilar Falcón ${ }^{(D)}$, Diego Hernán Zapattini ${ }^{(i)}$, Karina Elizabeth \\ Scavenius ${ }^{1}$, 2 , Alfredo Javier Meza (iD) 1,2 , Erdulfo Javier Galeano ${ }^{1}$, *Osmar \\ Antonio Centurión (iD) 1,2 \\ ${ }^{1}$ Universidad Nacional de Asunción, Hospital de Clínicas. División de Medicina \\ Cardiovascular. San Lorenzo, Paraguay. \\ ${ }^{2}$ Sanatorio Metropolitano, Departamento de Investigación en Ciencias de la Salud. \\ Fernando de la Mora. Paraguay.
}

Cómo referenciar este artículo/ How to reference this article:
Falcón $R$, Zapattini DH, Scavenius KE, Meza AJ, Galeano EJ, Centurión OA. Rol del bloqueo interauricular avanzado en la predicción de accidentes cerebrovasculares isquémicos. Mem. Inst. Investig. Cienc. Salud. 2021; 19(3): 105-114

\section{RE S U M E N}

El bloqueo interauricular (BIA) es un marcador significativo en la predicción del desarrollo de Fibrilación Auricular (FA). El sustrato histopatológico que se observa en el proceso de remodelación auricular es la fibrosis del miocardio auricular induciendo disincronía interauricular. La disfunción electromecánica de la aurícula izquierda (AI) produce una activación anormal de sus paredes, el aumento de la presión, la dilatación, la disfunción endotelial, y la fibrosis de la AI. Estas alteraciones favorecen la conducción lenta, el bloqueo unidireccional y el desarrollo de mecanismos de reentrada con la aparición de la FA con sus nefastas complicaciones, entre ellas el accidente cerebrovascular (ACV). El BIA está presente hasta en un $59 \%$ de los pacientes mayores de la población general y se asoció con un aumento del riesgo de unas 3 veces más de FA de nueva aparición y ACV isquémico. Es evidente el interés académico, clínico, y terapéutico en el diagnóstico electrocardiográfico certero del BIA avanzado, ya que el mismo se asocia con arritmias supraventriculares, fibrilación auricular, ACV embólicos y mortalidad. La detección de BIA avanzado en pacientes con ACV isquémico previo permite identificar a pacientes de alto riesgo de recurrencia en los que algunas terapias farmacológicas podrían ser beneficiosas. Los pacientes con BIA avanzado sin episodios previos de FA documentada también presentan un riesgo aumentado de ACV embólico. Por lo tanto, es necesario realizar ensayos clínicos randomizados cuyos resultados podrían avalar el uso de anticoagulantes en ausencia de FA documentada en pacientes con BIA avanzado.

Palabras clave: Bloqueo interauricular, fibrilación auricular, accidente cerebrovascular, embolismo.

\section{Role of advanced interatrial block in the prediction of ischemic strokes}

\footnotetext{
A B S T R A C T

Interatrial block (IAB) is a significant marker in the prediction of the development of Atrial Fibrillation (AF). The histopathological substrate observed in the atrial remodeling process is fibrosis of the atrial myocardium, inducing interatrial desynchrony. Electromechanical dysfunction of the left atrium (LA) produces abnormal

Fecha de recepción: Octubre 2021. Fecha de aceptación: noviembre 2021

*Autor correspondiente: Prof. Dr. Osmar Antonio Centurión, MD, PhD, FACC, FAHA.

Professor of Medicine. Asuncion National University (UNA). Department of Health Sciences Investigation.

Sanatorio Metropolitano. Teniente Ettiene 215 c/ Ruta Mariscal Estigarribia. Fernando de la Mora. Paraguay.

Email: osmarcenturion@hotmail.com
} 
activation of its walls, increased pressure, dilation, endothelial dysfunction, and fibrosis of the LA. These alterations favor slow conduction, unidirectional block and the development of reentry mechanisms with the appearance of AF with its disastrous complications, including cerebrovascular accident (CVA). IAB is present in up to $59 \%$ of older patients in the general population and was associated with a 3 -fold increased risk of new-onset AF and ischemic stroke. The academic, clinical, and therapeutic interest in the accurate electrocardiographic diagnosis of advanced IAB is evident, since it is associated with supraventricular arrhythmias, atrial fibrillation, embolic stroke and mortality. The detection of advanced IAB in patients with previous ischemic stroke allows the identification of patients at high risk of recurrence in which some pharmacological therapies could be beneficial. Patients with advanced IAB with no prior documented AF episodes are also at increased risk of embolic stroke. Therefore, it is necessary to conduct randomized clinical trials whose results might be available in the use of anticoagulants in the absence of documented AF in patients with advanced IAB.

Key words: Interatrial block, atrial fibrillation, stroke, embolism.

\section{INTRODUCCIÓN}

Se ha descrito que determinadas variables de la onda $P$, tales como las variaciones en morfología, voltaje, y duración, en el electrocardiograma (ECG) standard de 12 derivaciones permite identificar a los pacientes con riesgo de desarrollar arritmias auriculares, entre ellas la fibrilación auricular (FA) ${ }^{(1-5)}$. Además, se ha observado que ciertos parámetros electrocardiográficos han sido utilizados para estratificar a los pacientes y realizar un seguimiento más estricto de aquellos con alta probabilidad de desarrollar FA ${ }^{(2)}$.

Uno de estos parámetros electrocardiográficos arriba mencionados es el bloqueo interauricular (BIA) ${ }^{(6-11)}$. Esta alteración del ECG denota un retraso de conducción entre las aurículas derecha e izquierda a causa de un enlentecimiento en la conducción de impulsos a través del haz de Bachmann. El BIA es un marcador significativo en la predicción del desarrollo de FA ${ }^{(8,11-13)}$. El sustrato histopatológico que se observa en el proceso de remodelación auricular es la fibrosis del miocardio auricular induciendo disincronía interauricular. La disfunción electromecánica de la aurícula izquierda (AI) produce una activación anormal de sus paredes, el aumento de la presión, la dilatación, la disfunción endotelial, y la fibrosis de la AI ${ }^{(14-19)}$. Estas alteraciones fisiopatológicas favorecen la conducción lenta, el bloqueo unidireccional y el desarrollo de mecanismos de reentrada con la aparición de la FA con sus nefastas complicaciones, entre ellas el accidente cerebrovascular (ACV) ${ }^{(20-23)}$.

El BIA avanzado (Figura 1) se desarrolla debido a una conducción auricular totalmente bloqueada o parcialmente enlentecida a nivel del haz de Bachmann, lo que puede generar alteraciones en los períodos refractarios de la AI y la aparición de extrasístoles auriculares que podrían desarrollar arritmias auriculares, FA, y eventualmente embolias sistémicas y ACV ${ }^{(20-23)}$. Por lo tanto, es nuestra intención realizar un análisis detallado sobre del papel que posee el BIA en la detección y predicción de accidentes cerebrovasculares. Es fundamental poseer una mejor comprensión del BIA en ciertos pacientes con la predisposición clínica a desarrollar ACV y para refinar los criterios de selección diagnósticos y estrategias informativas para el manejo adecuado de la anticoagulación. 
Onda P normal

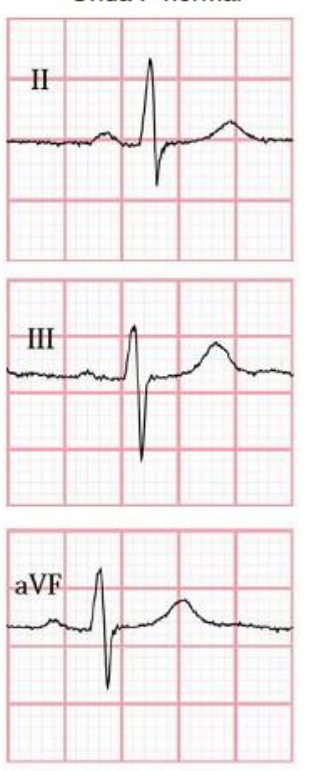

BIA-parcial
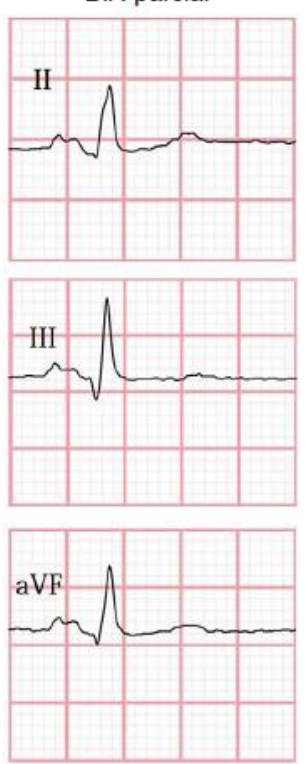

BIA-avanzado
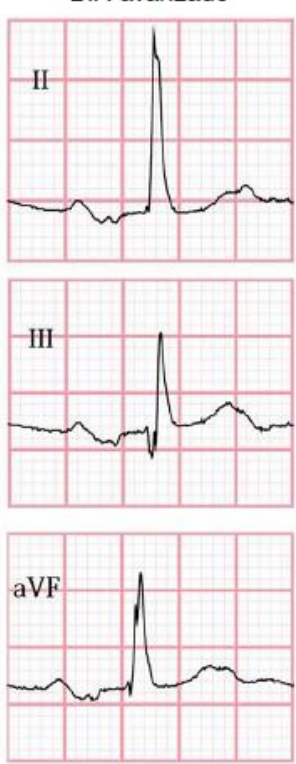

Figura 1. Ejemplos electrocardiográficos de una onda $P$ normal, y de Bloqueo interauricular (BIA) parcial y avanzado. El ejemplo del bloqueo interauricular avanzado tiene una morfología de patrón bifásico (positivo-negativo) con negatividad terminal en las derivaciones electrocardiográficas inferiores II, III, y aVF. Reimpreso con permiso de Carrillo-Loza K, Baranchuk A, Serrano F, Hassebb S, Espinosa Lirac F. Soriano E et al. El bloqueo interatrial avanzado predice recurrencia de infarto cerebral embólico de origen no determinado. Neurologia (Engl Ed) 2019; S0213-4853(19)30136-7. doi: 10.1016/j.nrl.2019.10.007. (24)

\section{Relación entre el BIA y los accidentes cerebrovasculares}

Es fundamental dar el énfasis necesario a la prevención y al diagnóstico precoz de enfermedades con gran impacto social, médico y económico a la salud pública como son la FA y los ACV isquémicos. Por lo tanto, es primordial intentar detectar la enfermedad en etapas tempranas de su evolución, en estadio sub-clínico, e identificar factores que determinen con gran confiabilidad la aparición y desarrollo de la enfermedad. El ECG convencional de 12 derivaciones es un método auxiliar de diagnóstico muy útil, de fácil disponibilidad, muy accesible para detectar cambios en el sistema de conducción cardíaca. El bloqueo interauricular que se produce por un bloqueo del haz de Bachmann es un marcador electrocardiográfico de arritmias auriculares, y se lo ha asociado clínicamente con un riesgo aumentado de FA e infarto cerebral (24-28). El haz de Bachmann está formado por un conjunto de fibras miocárdicas paralelas especializadas para la conducción de impulsos y representa el 80 a $85 \%$ de la conducción interauricular. Se encuentra ubicado en las paredes antero-superiores de ambas aurículas y su afectación parcial o total, da lugar al bloqueo interauricular, que puede ser: BIA parcial, si la conducción está retrasada de tal forma que en el ECG produce una onda $P \geq 120 \mathrm{~ms}$. Puede ser BIA avanzado si la conducción está del todo interrumpida y la despolarización auricular izquierda ocurre en dirección retrógrada caudo-craneal, por lo tanto la onda $\mathrm{P}$ es $\geq 120 \mathrm{~ms}$ y bifásica en las derivaciones inferiores II, III y aVF (Figura 2 ). 


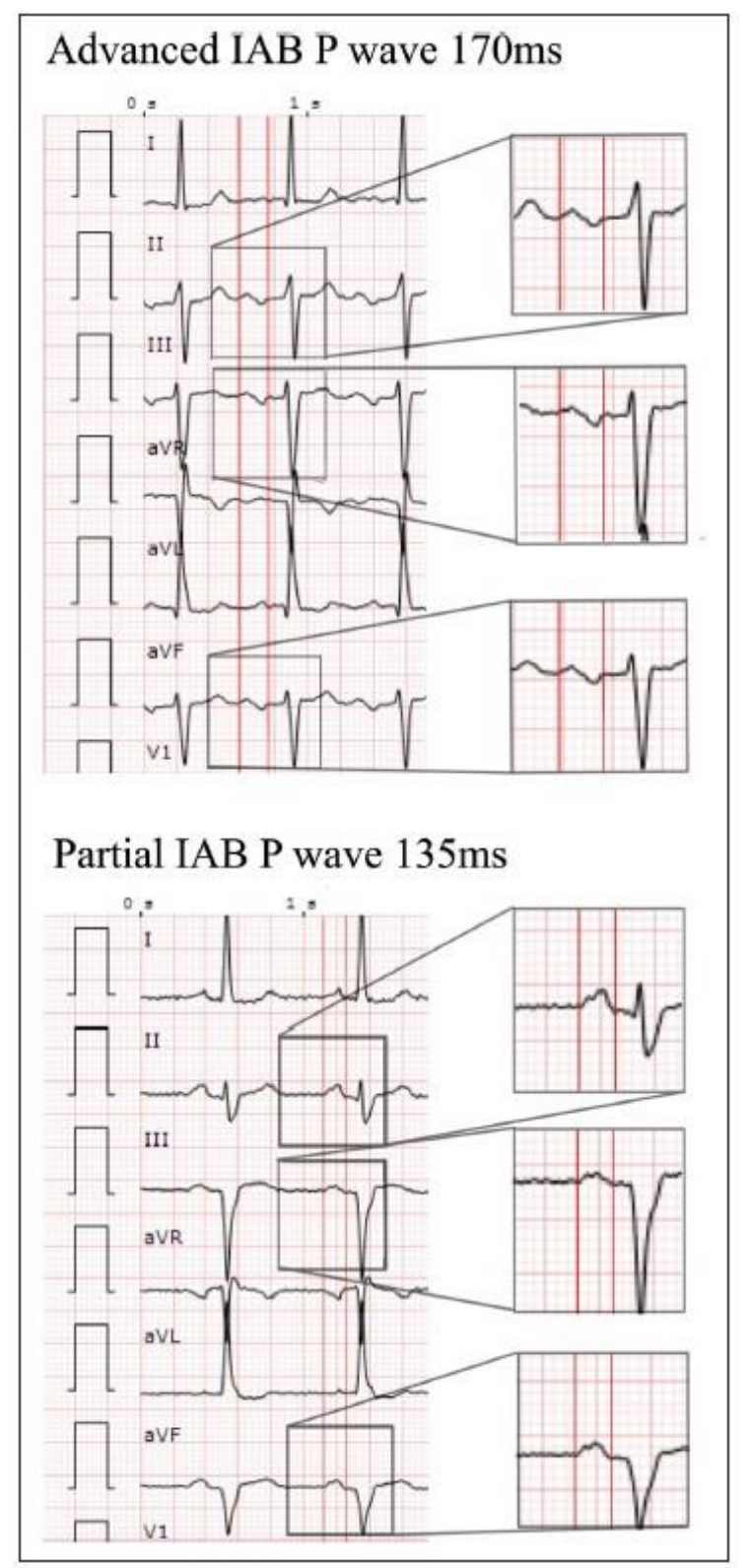

Figura 2. Ejemplos electrocardiográficos de Bloqueo interauricular (IAB) parcial y avanzado. Reimpreso con permiso de Vicent L, Fernandez-Cordon C, Nombela-Franco L, Escobar-Robledo LA, Ayesta A, Sole A, et al. Baseline ECG and Prognosis After Transcatheter Aortic Valve Implantation: The Role of Interatrial Block. J Am Heart Assoc. 2020; 9: e017624. DOI: 10.1161/JAHA.120.017624 (49)

Asad $\mathrm{N}$ y Spodik DH analizaron 1000 ECG no seleccionados, consecutivos, en pacientes hospitalizados, y observaron la presencia del BIA en el $47 \%$ de los pacientes que tenían ritmo sinusal normal durante la internación hospitalaria ${ }^{(26)}$. Por otro lado, Ninios I, et al. encontraron una prevalencia de BIA del $59 \%$ con una correlación significativa con la hipertensión arterial sistémica en un estudio realizado en la población general ambulatoria de una comunidad de ancianos mayores de 65 años ${ }^{(28)}$. Ariyarajah V, et al. observaron una prevalencia de BIA de $48,6 \%$ en un estudio realizado sobre una población de la comunidad de ancianos de un programa de atención integral para personas mayores (19). Agarwal $\mathrm{YK}$, et al. analizaron prospectivamente la prevalencia del BIA en un estudio con un seguimiento medio de 16 meses. La prevalencia de BIA fue comparada entre 308 pacientes que desarrollaron FA con un grupo control de 308 pacientes que permanecieron en ritmo sinusal durante el período de seguimiento. La prevalencia de BIA fue significativamente más alta (52\%) en los pacientes que desarrollaron FA, en comparación con solo un $18 \%$ en los pacientes controles ${ }^{(29)}$. Es evidente que el BIA es un hallazgo electrocardiográfico 
bastante frecuente, por ende, es importante buscarlo e identificarlo sobre todo en personas mayores. El sustrato anatómico del BIA avanzado es el de una miocardiopatía auricular fibrótica que desencadena una disincronía auricular que actúa como un mecanísmo desencadenante y de mantenimiento de la FA. Esta alteración de la arquitectura del miocardio auricular produce un remodelado auricular, estasis sanguínea e hipercoagulabilidad y la formación de trombos intra-auriculares ${ }^{(14-19)}$. Sin embargo, todavía no se ha demostrado el beneficio de la terapia de anticoagulación para los pacientes sin FA documentada. Por lo tanto, es recomendable una búsqueda proactiva de FA en los pacientes con BIA avanzado, ya que en este grupo de pacientes el sustrato anatómico y funcional de miocardiopatía auricular fibrótica con disincronía auricular, se traduce en una aurícula izquierda dilatada, hipocontractil que propicia el remodelado auricular y la favorece la estasis sanguínea y formación de trombos ${ }^{(14-19)}$.

Es importante notar que la asociación entre el BIA y los infartos cerebrales parece estar limitada a infartos no lacunares y se mantiene independientemente de la presencia de FA ${ }^{(28-31)}$. Existen evidencias clínicas que consideran a la cardiomiopatía auricular como un factor de riesgo independiente para ACV de tipo isquémico y que la existencia de trombos en la auricula izquierda ocurre independientemente del registro de FA ${ }^{(32-35)}$. En algunos estudios como los de $\mathrm{He} \mathrm{J}$, et al ${ }^{(13)}$. han demostrado que ciertas anormalidades de la onda $\mathrm{P}$, tales como la fuerza terminal de la onda $\mathrm{P}$ en la derivación $\mathrm{V} 1$, la duración de la onda $\mathrm{P}$, y el área máxima de la onda $\mathrm{P}$, predijeron episodios de ACV isquémico independientemente de la presencia de FA ${ }^{(13)}$. Debido a los cambios producidos por la remodelación auricular y su asociación con la dilatación auricular izquierda y con la disfunción auricular electromecánica, el BIA puede facilitar las alteraciones histológicas y electrofisiológicas y producir un sustrato anatómicoeléctrico para la formación de trombos intraauriculares y las embolias cerebrales y sistémicas ${ }^{(32)}$. En un estudio bien diseñado, Lorbar $M$, et al ${ }^{(3)}$. estudiaron en forma retrospectiva a 104 pacientes en ritmo sinusal con accidentes cerebrovasculares embólicos y observaron que el BIA estaba presente en un $80 \%$ de estos pacientes ${ }^{(3)}$. En otro estudio retrospectivo, Ariyarajah $\mathrm{V}$, et al ${ }^{(20)}$. compararon 85 pacientes con ACV embólico con 208 pacientes con ACV no embólico, que fueron emparejados por factores de riesgo de accidente cerebrovascular. Este estudio demostró que un $88 \%$ de los pacientes estaban en ritmo sinusal, pero la prevalencia de BIA fue significativamente mayor en los pacientes con ACV embólico $(60.6 \%)$ en comparación con los pacientes que tenían ACV no embólico $(39.8 \%){ }^{(20)}$. Los factores de riesgo cardiovascular para el BIA avanzado, la FA y el ACV embólico parecen ser muy similares, y la patogénesis subyacente probablemente se deba a fibrosis miocárdica y remodelación auricular ${ }^{(14-19)}$. El BIA avanzado produce una disfunción eléctrica y mecánica auricular que propicia el desarrollo de una miocardiopatía fibrótica auricular que ha sido muy bien descrita por un consenso de expertos ${ }^{(32)}$. La fibrosis favorece la estasis sanguínea y la trombosis intrauricular, principalmente en la orejuela, así como también favorece el desarrollo de arritmias supraventriculares, especialmente la FA (33). El tratamiento que demostró categóricamente una mejoría de la sobrevida en pacientes con FA fue la prevención del ACV embólico mediante la anticoagulación (32). De hecho, un $20 \%$ de los episodios de ACV embólico se atribuyen a la FA, aumentando así al doble la tasa de mortalidad y la tasa de un nuevo ACV en cinco veces ${ }^{(36-39)}$. Durante el tratamiento preventivo del ACV embólico, la escala CHA2DS2VASc, ha sido ampliamente validada para decidir la anticoagulación en la población general y de esta manera prevenir la aparición de eventos tromboembólicos ${ }^{(40-50)}$. Por otro lado, la escala HASBLED valora el riesgo de hemorragias. El balance adecuado entre ambas escalas determina en gran medida el éxito terapéutico y la seguridad de la anticoagulación. Teniendo en cuenta los efectos adversos de la anticoagulación, es de vital importancia estandarizar la indicación de la misma basándose en criterios validados en estudios previos con alto nivel de confiabilidad ${ }^{(41)}$.

\section{Estudios clínicos sobre el BIA y los ACV embólicos}

La FA era considerada como la causa común final que originaba las tromboembolias sistémicas y cerebrales. De hecho, se ha observado una asociación entre FA y ACV isquémico en un estudio reciente ${ }^{(48)}$ con seguimiento a 10 años obtenidos al agregar el bloqueo interauricular a los modelos de factores de riesgo convencionales (Figura 
3). Sin embargo, se ha demostrado que existe una ausencia de una relación temporal de los episodios paroxísticos de FA con la aparición de ACV embólicos ${ }^{(46-49)}$. Por esta razón la fibrosis miocárdica auricular adquiere una relevancia muy importante por su relación con el desarrollo de estasis sanguínea en las aurículas y orejuelas auriculares que favorece la formación de trombos intracavitarios. Es entendible y evidente el énfasis preponderante que se ha puesto en los medios auxiliares de diagnóstico por imágenes actualmente como medio de diagnóstico de fibrosis auricular. La resonancia magnética nuclear cardiaca es el gold standard para detectar la miocardiopatía auricular fibrótica $(15,44)$, que es el sustrato anatomopatológico de la mayoría de los casos de pacientes con BIA avanzado y de FA. Incluso se ha demostrado que los pacientes con BIA avanzado pueden presentar un alto grado de fibrosis del miocardio auricular sin FA documentada ${ }^{(15)}$. Por otro lado, la ecocardiografía con la técnica de speckle-tracking también permite valorar adecuadamente la fibrosis auricular y tiene implicaciones no solamente diagnósticas sino pronósticas en lo que respecta a las recurrencias de los episodios de FA $(17,23,45)$.

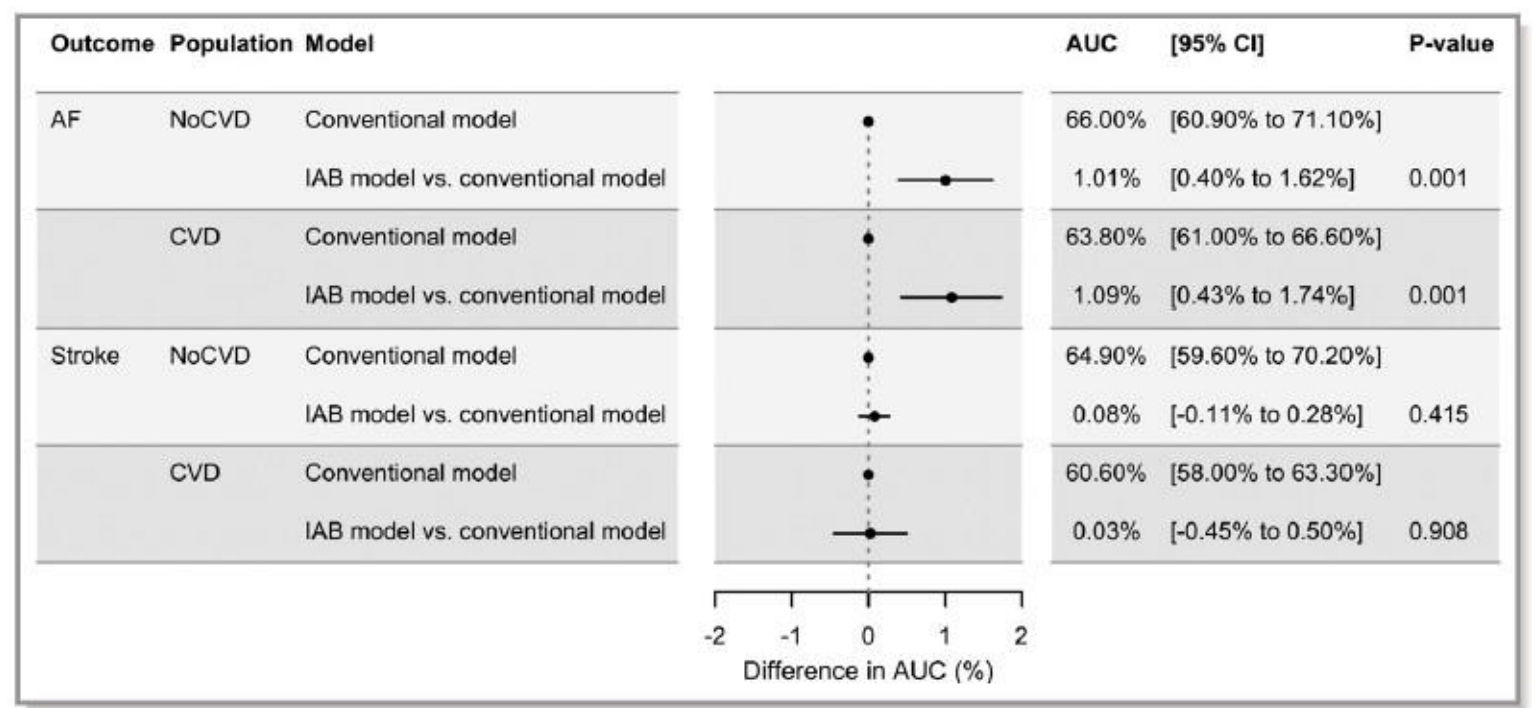

Figura 3. Diferencias en el área bajo la curva para los resultados a 10 años de fibrilación auricular y ACV isquémico obtenidos al agregar el bloqueo interauricular a los modelos de riesgo convencionales de fibrilación auricular y ACV isquémico, respectivamente, estratificados por la presencia o ausencia de enfermedades cardiovasculares al inicio del estudio. AF indica fibrilación auricular; AUC, área bajo la curva; IC: intervalo de confianza; CVD: enfermedad cardiovascular al inicio del estudio; IAB, bloqueo interauricular; sin CVD, sin enfermedad cardiovascular al inicio del estudio. Reimpreso con permiso de Skov MW, Ghouse J, Kuhl JT, Pyotr G, Platonov PG, Graff C, Fuchs A. Risk Prediction of Atrial Fibrillation Based on Electrocardiographic Interatrial Block J Am Heart Assoc. 2018; 7: e008247. DOI: 10.1161/JAHA.117.008247.

El estudio ARIC ha servido como fuente de análisis para investigar la relación existente entre el BIA y los ACV isquémicos. En este contexto, O'Neal et al (27). examinaron la asociación entre BIA y la incidencia de infarto cerebral (27). Ellos demostraron que el BIA fue un factor de riesgo independiente para el ACV isquémico. Se estima que en USA, ocurren aproximadamente 700.000 nuevos casos de ACV isquémico por año y no se ha determinado aún cuál es el mejor tratamiento de prevención secundaria $(28,29)$. Los resultados obtenidos por dos estudios que investigaron la recurrencia de infarto cerebral en pacientes con ACV isquémico tratados con ACO no han sido satisfactorios. Por un lado, el estudio ESUS NAVIGATE, que comparó $15 \mathrm{mg}$ de rivaroxaban contra $100 \mathrm{mg}$ de aspirina; y por el otro, el estudio RESPECT ESUS, que comparó dos dosis de dabigatrán (100 y150 mg dos veces por día) contra la aspirina. Estos dos ensayos clínicos fallaron en demostrar la eficacia de los ACO en la prevención de recurrencia de infarto cerebral en este grupo de pacientes. Además, en el caso del rivaroxaban, se observó la asociación con un mayor riesgo de hemorragia ${ }^{(30,31)}$. Un dato muy interesante a tener en cuenta es que ambos grupos de tratamiento mostraron una tasa elevada de recurrencia en el estudio 
ESUS NAVIGATE. Este hecho singular pone de relieve la necesidad de una mejor selección de pacientes y una mejor opción terapéutica.

Por otro lado, Carrillo-Loza k, et al. ${ }^{(24)}$ investigaron al BIA avanzado como predictor de recurrencia de ACV embólico en pacientes con infarto cerebral embólico de origen no determinado (ESUS). Los autores analizaron los datos clínicos, las características de la onda P y presencia de BIA en el electrocardiograma de 104 pacientes con diagnóstico confirmado de ESUS con un seguimiento medio de 15 meses. La recurrencia de ESUS fue el desenlace primario. Se encontró BIA en 36 pacientes (34,6\%), de los cuales tenían BIA parcial en $29(27,9 \%)$, y BIA avanzado en $7(6,7 \%)$ pacientes. Un total de 16 pacientes $(15,4 \%)$ presentaron ACV recurrente; de los cuales 5 tenían BIA parcial, y 4 tenían BIA avanzado $(p=0,01)$. La duración media de la onda $\mathrm{P}$ fue mayor en pacientes con recurrencia $(p=0,009){ }^{(24)}$. En el análisis multivariado de regresión logística, los factores de riesgo independientes para recurrencia de ACV embólico fueron: el BIA-a $(p<0,001)$, el género masculino $(p=0,028)$ y la edad mayor a 50 años $(p=0,039)$. Por lo tanto, los autores concluyeron que el BIA avanzado y una edad mayor a 50 años predicen la recurrencia de ACV embólico ${ }^{(24)}$. Estos hallazgos sugieren que la detección de BIA avanzado en pacientes con ACV isquémico previo permite identificar a pacientes de alto riesgo de recurrencia en los que algunas terapias farmacológicas podrían ser beneficiosas.

En un estudio descriptivo, observacional y retrospectivo fueron analizadas las características clínicas, eléctricas y ecocardiográficas de pacientes portadores de BIA. La población estudiada conformó una muestra total de 165 pacientes consecutivos, que eran mayores de edad, se hallaban en ritmo sinusal y tuvieron un seguimiento a largo plazo durante tres años ${ }^{(35)}$. Se diagnosticaron 63 casos de BIA avanzado en el ECG y por lo tanto se seleccionó una muestra similar de pacientes consecutivos con BIA parcial y sin BIA que conformó una muestra total de 165 pacientes para el estudio. Los autores observaron que los factores vinculados con riesgo de padecer un BIA avanzado son tener $\geq 65$ años, diabetes, disfunción diastólica del ventrículo izquierdo y dilatación de la aurícula izquierda (35). Además, observaron que los pacientes con BIA avanzado poseen una mayor tasa de ACV isquémico que aparece junto con antecedente de FA o sin ella. Estos resultados destacan la posibilidad de realizar tamizado clínico y prevención de la FA y la necesidad de individualizar y precisar si los enfermos con BIA avanzado deben anticoagularse a fin de prevenir los ACV isquémicos. El ensayo clínico randomizado "AtRial Cardiopathy and Antithrombotic Drugs In Prevention After Cryptogenic Stroke", se encuentra actualmente en pleno desarrollo e incluye pacientes con ACV isquémico de origen indeterminado y cardiomiopatía auricular que están recibiendo ya sea apixaban o aspirina ${ }^{(34)}$. Existe mucho interés en los resultados de este ensayo clínico ya que los hallazgos podrían aclarar si el alto riesgo de recurrencia en pacientes con ACV isquémico de origen indeterminado puede ser reducido con otras alternativas de terapia de anticoagulación.

\section{CONCLUSIÓN}

Es evidente el interés académico, clínico, y terapéutico en el diagnóstico electrocardiográfico certero del BIA avanzado, ya que el mismo se asocia con arritmias supraventriculares, fibrilación auricular, ACV embólicos y mortalidad. La detección de BIA avanzado en pacientes con ACV isquémico previo permite identificar a pacientes de alto riesgo de recurrencia en los que algunas terapias farmacológicas podrían ser beneficiosas. Los pacientes con BIA avanzado sin episodios previos de FA documentada también presentan un riesgo aumentado de ACV embólico. Por lo tanto, es necesario realizar ensayos clínicos randomizados cuyos resultados podrían avalar el uso de anticoagulantes en ausencia de FA documentada en pacientes con BIA avanzado.

Fuentes de financiación: Ninguna.

Declaración de conflicto de intereses: Ninguno. 


\section{Contribución de autores:}

Todos los autores han contribuido en la elaboración del presente manuscrito.

\section{REFERENCIAS BIBLIOGRÁFICAS}

1. Alexander B, Milden J, Hazim B, Haseeb $S$, Bayes-Genis A, Elosua R, et al. New electrocardiographic score for the prediction of atrial fibrillation: The MVP ECG risk score (morphology-voltage-Pwave duration). Ann Noninvasive Electrocardiol. 2019; 24(6): 1-7. Doi: 10.1111/anec.12669

2. Goyal SB, Spodick DH. Electromechanical dysfunction of the left atrium associated with interatrial block. Am Heart J. 2001 Nov; 142(5): 823-7. Doi:

10.1067/mhj.2001.118110

3. Lorbar $M$, Levrault $R$, Phadke JG, Spodick $\mathrm{DH}$. Interatrial block as a predictor of embolic stroke. Doi: Am J Cardiol 2005; 95(5): 667-8. Doi: 10.1016/j.amjcard.2004.10.059

4. Conde D, Baranchuk A. Interatrial block as anatomical-electrical substrate for supraventricular arrhythmias: Báyes Syndrome. Arch Cardiol Mex. 2014; 84(1): 32-40. Doi:

10.1016/j.acmx.2013.10.004

5. Bacharova L, Wagner GS. The time for naming the interatrial block syndrome: Bayes' Syndrome. J Electrocardiol 2015; 48: 133-4.

Doi:

10.1016/j.jelectrocard.2014.12.022

6. Bayés de Luna $A$, Cladellas $M$, Oter $R$, Torner P, Guindo J, Martí V, et al. Interatrial conduction block and retrograde activation of the left atrium and paroxysmal supraventricular tachyarrhythmia. Eur Heart J 1988; 9: 1112-8. Doi:

10.1093/oxfordjournals.eurheartj.a0624 $\underline{07}$

7. Bayes de Luna A, Fort de Ribot R, Trilla E, Julia J, Garcia J, Sadurni J, et al. Electrocardiographic and vectorcardiographic study of interatrial conduction disturbances with left atrial retrograde activation. J Electrocardiol 1985; 18(1):1-13. Doi: $10.1016 /$ s00220736(85)80029-7

8. Bayés de Luna $A$, Oter $M C$, Guindo J. Interatrial conduction block with retrograde activation of the left atrium and paroxysmal syupraventricular tachyarrhythmias: influence of preventive antiarrhythmic treatment. Int J Cardiol 1989; 22(2): 147-50. Doi: 10.1016/0167-5273(89)90061-2

9. Falcón Fleytas RP, Scavenius Aguilera $\mathrm{KE}$, Meza AJ, Centurión OA. Advanced interatrial block in patients with systemic arterial hypertension and atrial fibrillation. Mem. Inst. Investig. Cienc.
Salud. 2020; 18(2): 74-85. Doi: $10.18004 / \mathrm{mem} . \mathrm{iics} / 1812-$ 9528/2020.018.02.74

10. Centurión OA. Role of interatrial block recognition: A closer look to the Bayés syndrome. J Atr Fibrillation 2019; 12(2):2246. doi: 10.4022/jafib.2246.

11. Baranchuk $A$, Enriquez $A$, Antiperovitch $P$, Alexander B, Çinier G. Advanced interatrial block as a key marker for atrial fibrillation recurrence: Bayés' syndrome. J Geriatr Cardiol. 2017; 14 (3):169-73. Doi: 10.11909/j.issn.16715411.2017.03.005

12. Tse G, Wong CW, Gong $M$, Wong WT, Bazoukis G, Wong $\mathrm{SH}$, et al. International Health Informatics Study (IHIS) Network. Predictive value of inter-atrial block for new onset or recurrent atrial fibrillation: A systematic review and meta-analysis. Int J Cardiol 2018; 250: 152-6. Doi: 10.1016/j.ijcard.2017.09.176

13. He J, Tse G, Korantzopoulos $P$, Letsas $\mathrm{KP}$, Ali-Hasan-Al-Saegh $\mathrm{S}$, Kamel $\mathrm{H}$, et al. P-Wave Indices and Risk of Ischemic Stroke: A Systematic Review and MetaAnalysis. Stroke. 2017; 48(8): 206672. Doi:

10.1161/STROKEAHA.117.017293

14. Ciuffo L, Bruña $V$, Martínez-Sellés $M$, Doria de Vasconcellos $\mathrm{H}$, Tao S, Zghaib $\mathrm{T}$, et al. Association Between Interatrial Block, Left Atrial Fibrosis and Mechanical Dyssynchrony: Electrocardiography-Magnetic

Resonance Imaging Correlation. J Cardiovasc Electrophysiol. 2020 Jul; 31(7): 1719-25. Doi:

10.1111/jce. 14608 .

15. Benito EM, De Luna $A B$, Baranchuk $A$, Mont L. Extensive atrial fibrosis assessed by late gadolinium enhancement cardiovascular magnetic resonance associated with advanced interatrial block electrocardiogram pattern. Europace 2017; 19(3):377. Doi: 10.1093/europace/euw294

16. Saremi F, Channual S, Krishnan S, Gurudevan SV, Narula J, Abolhoda A. Bachmann Bundle and its arterial supply: imaging with multidetector CT-implications for interatrial conduction abnormalities and arrhythmias. Radiology 2008; 248(2): 447-57. Doi: 10.1148/radiol.2482071908

17. Lacalzada-Almeida J, Izquierdo-Gómez MM, Belleyo-Belkasem C, BarrioMartínez P, García-Niebla J, Elosua R, et al. Interatrial block and atrial 
remodeling assessed using speckle tracking echocardiography. BMC Cardiovasc. Disord 2018; 18(1): 38. doi: $10.1186 / \mathrm{s} 12872-018-0776-6$

18. Goyal SB, Spodick DH. Electromechanical dysfunction of the left atrium associated with interatrial block. Am. Heart J 2001; 142(5): 8237. Doi: $10.1067 / \mathrm{mhj} .2001 .118110$

19. Ariyarajah $V$, Puri $P$, Kranis $M$, Wilner DA, Spodick DH. Prevalence of interatrial block in the Program of AllInclusive Care for the Elderly (PACE). Am J Geriatr Cardiol 2006; 15(3): 1747. Doi: 10.1111/j.1076-7460.2006.04518.x

20. Ariyarajah V, Puri P, Apiyasawat S, Spodick DH. Interatrial block: a novel risk factor for embolic stroke? Ann Noninvasive Electrocardiol 2007; 12(1): 15-20. Doi:

10.1111/j.1542-474X.2007.00133.X

21. Ciuffo $L$, Inoue $Y Y$, Tao $S$, Gucuk Ipek $E$, Balouch M, Lima JAC, et al. Mechanical dyssynchrony of the left atrium during sinus rhythm is associated with history of stroke in patients with atrial fibrillation. Eur. Heart J. Cardiovasc. Imaging 2018; 19(4):433-41. Doi: 10.1093/ehjci/jex156

22. Baranchuk A, Yeung C. Advanced interatrial block predicts atrial fibrillation recurrence across different populations: Learning Bayes syndrome. Int. J. Cardiol 2018; 272: 221-2. Doi: 10.1016/j.ijcard.2018.08.025

23. Lacalzada-Almeida J, Izquierdo-Gomez MM, Garcia-Niebla J, Elosua R, JiménezSosa A, Baranchuk A, et al. Advanced interatrial block is a surrogate for left atrial strain reduction which predicts atrial fibrillation and stroke. Ann. Noninvasive Electrocardiol 2019: 24(4): e12632. doi: 10.1111/anec.12632.

24. Carrillo-Loza K, Baranchuk A, Serrano F, Hassebb S, Espinosa Lirac F. Soriano $E$ et al. El bloqueo interatrial avanzado predice recurrencia de infarto cerebral embólico de origen no determinado. Neurologia (Engl Ed) 2019; S02134853(19)30136-7. Doi: 10.1016/j.nrl.2019.10.007.

25. Escobar-Robledo LA, Bayés-de-Luna $A$, Lupon J, Baranchuk A, Moliner P, Martínez-Sellés $M$, et al. Advanced interatrial blockpredicts new-onset atrial fibrillation and ischemic stroke in patients with heart failure: "The Bayes'Syndrome-HF" Study. Int J Cardiol. 2018; 271:174-80.

26. Asad N, Spodick DH. Prevalence of interatrial block in a general hospital population. Am J Cardiol 2003; 2003; 91: 609-10.
27. O'Neal WT, Kamel $\mathrm{H}$, Zhang ZM, Chen LY, Alonso A, Soliman EZ. Advanced interatrial block and ischemic stroke: The atherosclerosis risk in communities study. Neurology. 2016; 87:352-6.

28. Ninios I, Pliakos C, Ninios V, Karvounis $H$, Louridas $G$. Prevalence of interatrial block in a general population of elderly people. Ann Noninvasive Electrocardiol 2007; 12: 298-300.

29. Agarwal YK, Aronow WS, Levy JA, Spodick DH. Association of interatrial block with development of atrial fibrillation. Am J Cardiol 2003; 91: 882.

30. Hart RG, Sharma M, Mundl H, Kasner S, Bangdiwala SI, Ber-kowitz SD, et al. Rivaroxaban for stroke prevention afterembolic stroke of undetermined source. N Engl J Med. 2018; 378: 2191201.

31. Diener HC, Easton JD, Granger CB, Cronin L, Duffy C, CottonD, et al. RESPECT ESUS Investigators. Design of Randomized, double-blind, Evaluation in secondary Stroke Prevention comparing the EfficaCy and safety of the oral Thrombin inhibitor dabigatran etexilate vs. acetylsalicylic acid in patients withEmbolic Stroke of Undetermined Source (RE-SPECT ESUS). Int J Stroke. 2015; 10:1309-12.

32. Goette A, Kalman JM, Aguinaga L, Akar J Cabrera JA, Chen SA, et al. EHRA/HRS/APHRS/SOLAECE expert consensus on atrial cardiomyopathies: definition, characterization, and clinical implication. Europace 2016; 18:14551490 doi: 10.1093/europace/euw161.

33. Hirsh B, Copeland-Halperin R, Halperin J. Fibrotic atrial cardiomyopathy, atrial fibrillation, and thromboembolism: mechanistic links and clinical inferences. ] Am Coll Cadiol. 2015; 65(20): 2239225.

34. Kamel $\mathrm{H}$, Longstreth WT Jr, Tirschwell DL, Kronmal RA, Bro-derick JP, Palesch $Y Y$, et al. The AtRial Cardiopathy andAntithrombotic Drugs In prevention After cryptogenic strokerandomized trial: Rationale and methods. Int J Stroke. 2019; 14(2): 207-14. Doi: $10.1177 / 1747493018799981$

35. Gentille-Lorente D, Salvadó-Usach T. Clinical, electrical and echocardiographic characteristics of patients with advanced interatrial block. Arch Cardiol Mex. 2021; 91(1):135-8. Doi: 10.24875/acm.20000239

36. Giner-Soriano M, Casajuana M, RosoLlorach A, Vedia C, Morros R. Effectiveness, safety and costs of stroke prevention in non-valvular auricular fibrillation. Study of cohorts matched by Propensity score. Aten Primaria. 2020; 
52(3): 176-84. Doi: 10.1016/j.aprim.2019.06.002

37. Belmar Vega L, de Francisco ALM, Bada da Silva J, Galván Espinoza L, Fernández Fresnedo G. Nuevos anticoagulantes orales en pacientes con enfermedad renal crónica. Nefrología. 2017; 37(3): 229-356. Doi: 10.1016/j.nefro.2016.08.006

38. Sánchez Soriano RM, Albero Molina MD, Chamorro Fernández CI, Juliá-Sanchís $\mathrm{R}$, López Menchero $\mathrm{R}$, Del Pozo Fernández $C$, et al. Impacto pronóstico a largo plazo de la anticoagulación en pacientes en hemodiálisis con fibrilación auricular. Nefrologia. 2018; 38(4): 394-400. Doi: https://doi.org/10.1016/j.nefro.2017.11 .026

39. De Simón AF, Coll-Vinent $B$, Martín A, Suero C, Sánchez J, Varona $M$, et al. Cardioversion in recent onset atrial fibrillation. Emergencias. 2019; 31(4): 227-33. Disponible en: https://pubmed.ncbi.nlm.nih.gov/31347 801/

40. White JL, Hollander JE. Fibrilación auricular rápida, cardioversión rápida, regreso a casa rápido. Emergencias. 2019; 31: 223-4. Disponible en: https://medes.com/publication/144897

41. del Arco Galán C. Medicamentos, fibrilación auricular permanente y problemas derivados. Emergencias. 2016; 28(2): 71-2.

42. De Simón AF, Coll-Vinent $B$, Martín A, Suero C, Sánchez J, Varona $M$, et al. Cardioversion in recent onset atrial fibrillation. Emergencias. 2019; 31(4):227-33.

43. White JL, Hollander JE. Fibrilación auricular rápida, cardioversión rápida, regreso a casa rápido. Emergencias. 2019; 31: 223-4.

44. Marrouche NF, Wilber D, Hindricks G, et al. Association of atrial tissue fibrosis identified by delayed enhancement MRI and atrial fibrillation catheter ablation. The DECAAF study. JAMA. 2014; 311: 498-506. Doi: 10.1001/jama.2014.3

45. Montserrat S, Gabrielli L, Bijnens B, et al. Left atrial deformation predicts success of first and second percutaneous atrial fibrillation ablation. Heart Rhythm. 2015; 12: 11-18. Doi: 10.1016/j.hrthm.2014.08.032

46. Martin DT, Bersohn MM, Waldo $A L$, et al. IMPACT Investigators. Randomized trial of atrial arrhythmia monitoring to guide anticoagulation in patients with implanted defibrillator and cardiac resynchronization devices. Eur Heart J. 2015; 36:1660-8. Doi:

10.1093/eurheartj/ehv115

47. Bisbal F, Baranchuk A, Braunwald E, Bayés de Luna A, Bayés-Genís A. Atrial failure as a clinical entity. JACC Review Topic of the Week. J Am Coll Cardiol. 2020; 75(2): 222-32. Doi: 10.1016/j.jacc.2019.11.013

48. Skov MW, Ghouse J, Kuhl JT, Pyotr G, Platonov PG, Graff C, Fuchs A, et al.

Risk Prediction of Atrial Fibrillation Based on Electrocardiographic Interatrial Block J Am Heart Assoc. 2018;7:e008247. Doi: 10.1161/JAHA.117.008247

49. Vicent L, Fernandez-Cordon C, NombelaFranco L, Escobar-Robledo LA, Ayesta A, Sole $A$, et al. Baseline ECG and Prognosis After Transcatheter Aortic Valve Implantation: The Role of Interatrial Block. J Am Heart Assoc. 2020; 9: e017624. DOI: 10.1161/JAHA.120.017624.

50. Bayés de Luna $A$, Baranchuk $A$, Escobar Robledo LA, Massó van Roessel A, Martínez Sellés $M$. Diagnosis of interatrial block. J Geriatric Cardiol 2017; 14: 161-65. doi: 10.11909/j.issn.1671-5411.2017.03.007 